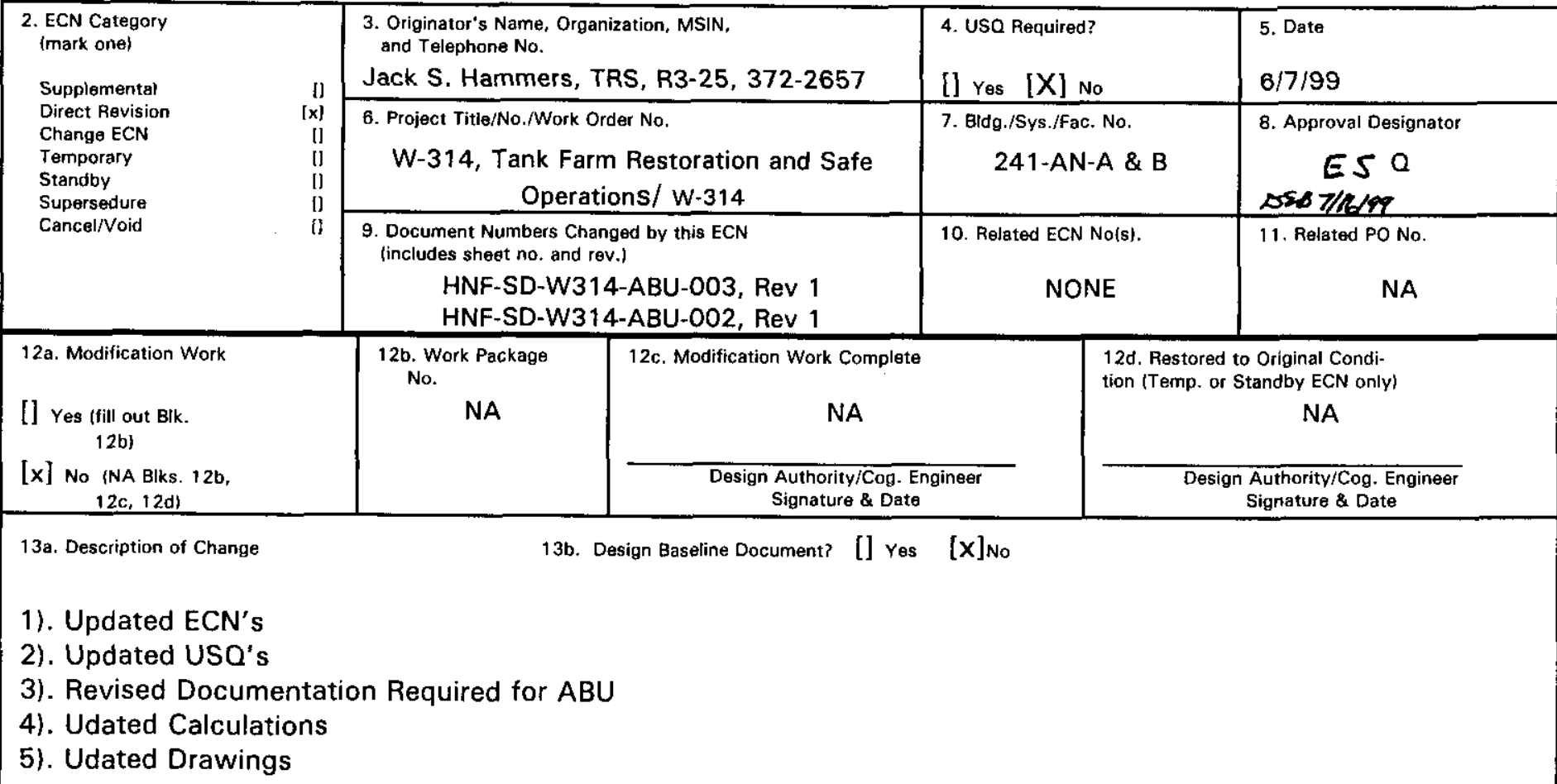

\begin{tabular}{|c|c|c|c|c|c|c|c|}
\hline Criteria Change & [] & Design Improvement & {$[x]$} & Environmental & [] & Facility Deactivation & [] \\
\hline As-Found & {$[x]$} & Facilitate Const & [] & Const. Error/Omission & [] & Design Errar/Omission & [] \\
\hline
\end{tabular}

14b. Justification Details

Updated ABU's to reflect current Project status.

"This modificatioin will not change collective dose sinec it has no impact on radiological sources,

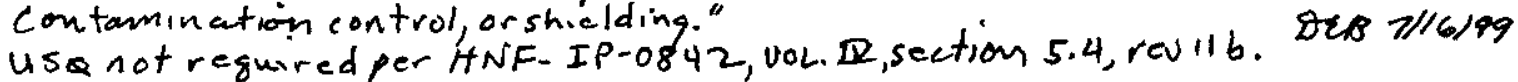

15. Distribution (include name, MSIN, and no. of copies)

RELEASE STAMP

See attached Distribution Sheet 


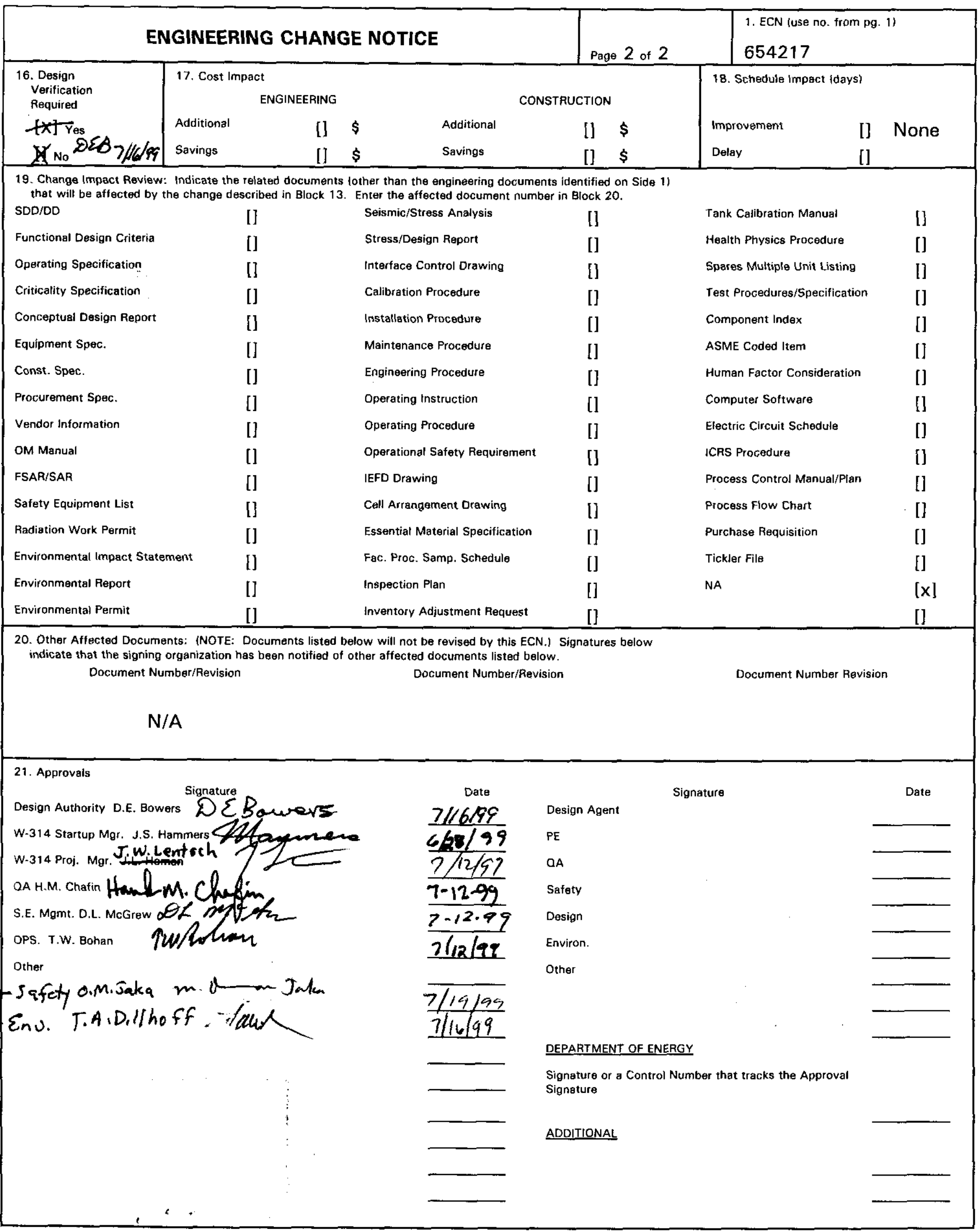




\section{Project W-314 241-AN-A Valve Pit Upgrade Acceptance For Beneficial Use}

Jack S. Hammers

Numatec Hanford Company. Richland, WA 99352

U.S. Department of Energy Contract DE-AC06-96RL13200

EDT/ECN: 6542187 94i-199 UC: 620

Org Code: 408

B\&R Code: EW3130010

Charge Code: 106048

Total Pages: -278022

Key Words: AN-A Vaive Pit Upgrade

Abstract: N/A

TRADEMARK DISCLAIMER. Reference herein to any specific commercial product, process, or service by trade name. trademark, manufacturer. or otherwise, does not necessarily constitute or imply its endorsement, recormendation. or favoring by the United States Government or any agency thereof or its contractors or subcontractors.

Printed in the United States of America. To obtain copies of this document. contact: Document Control Services. P.0. Box 950. Mailstop H6-08, Richland WA 99352. Phone (509) 372-2420: Fax (509) 376-4989.

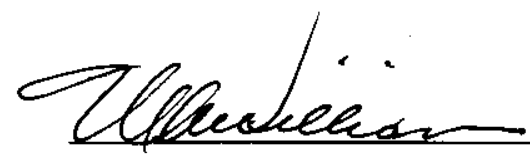

Release Approva?

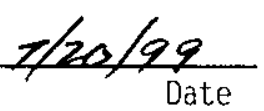

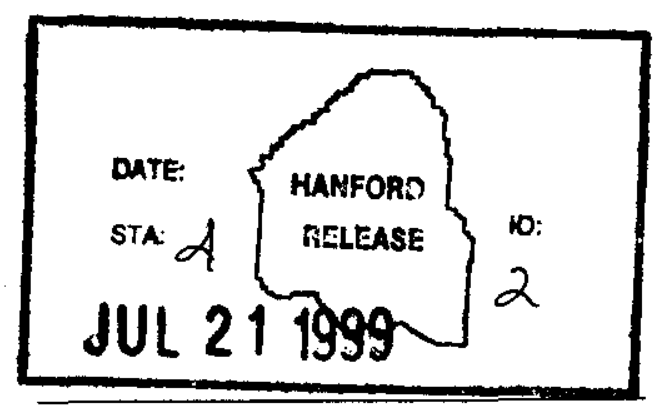

Release Stamp

\section{Approved for Public Release}




\section{RECORD OF REVISION}

(1) Document Number

HNF -SD-W314-ABU-

(2) Title

Project W-314 241-AN-A Valve Pit Upgrade. Acceptance for Beneficial USe

CHANGE CONTROL RECORD

(3)

Revision

0

1

ECN 650503. HNF-SD-W314-ABU-002. Update responsibjlity matrix and $A B U$ Index

2

ECN 654218?. HNF-SO-W314-ABU-002, Rev 2,

Rs

(4) Description of Change - Replace, Add, and Delete Pages

(7) EDT-618918, Dated August 29, 1997

Update responsibility matrix and $A B U$ Index
Authorized for Release

(5) $\operatorname{Cog}$. Engr.

(6) Cog. Mgr. Date

TL Warnick WH Hays

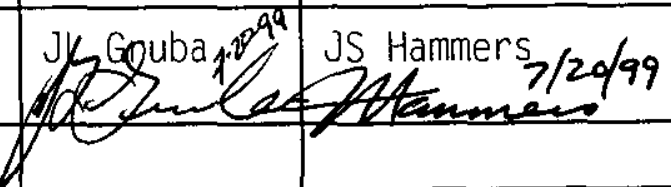


Program/Project Title: Project W-314, Tank Farm Restoration and Safe Operation, Phase 1

Component/System: $\quad$ AN-A Valve Pit Upgrades

June 28, 1999

\section{PROJECT W-314 \\ 241-AN-A VALVE PIT UPGRADE ACCEPTANCE FOR BENEFICIAL USE}


This listing identifies the responsibilities and requirements, applicable to the 241-AN-A Valve Pit Upgrades portion of Project W-314, for Acceptance for Beneficial Use in accordance with HNF-IP-0842, Vol IV, Sec 3.12.

At project turnover, the end user accepts the affected Structures, Systems, and Components (SSCs) for beneficial use. This checklist is used to help the end user ensure that all documentation, training, and testing requirements are met prior to turnover.

This checklist specifically identifies those items related to the upgrading of the 241-AN-A valve pit. The upgrades include: the installation of jumper/valve manifolds with position sensors, replacement pit leak detection systems, construction of replacement cover blocks, and electrical upgrades to support the instrumentation upgrades. 
Program/Project Title: Project W-314. Tank Farm Restoration and Safe Operation, Phase 1 Component/System: AN-A Valve Pit Upgrades June 28,1999

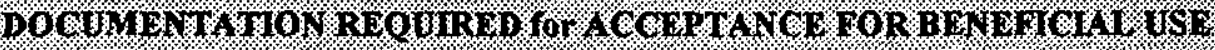

\begin{tabular}{|c|c|c|c|}
\hline DESCRIPTION & RESPONSIBILITY & DESCRIPTION & RESPONSIBILITY \\
\hline 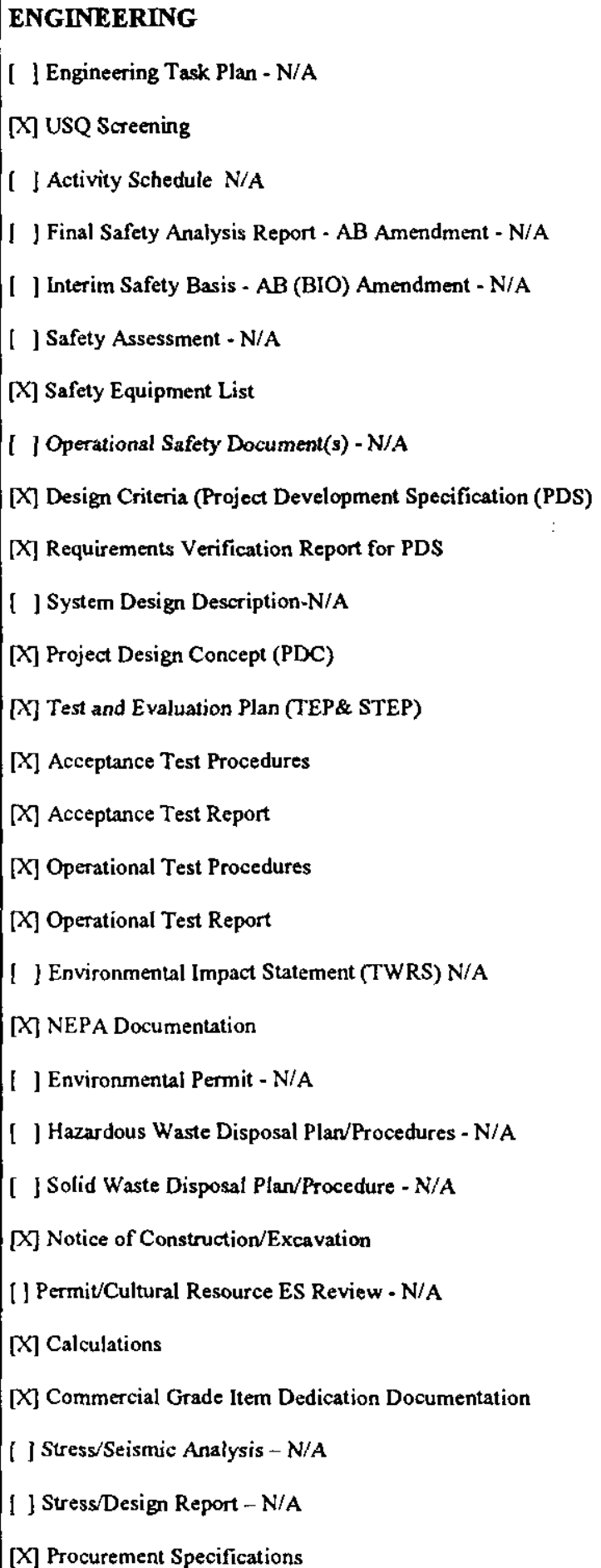 & $\begin{array}{l}\text { S.H. Rifaey } \\
\text { M.A. Lane } \\
\text { P.J. Bedell } \\
\text { P.J. Bedell } \\
\text { J.S. Hammers } \\
\text { J.S. Hammers } \\
\text { J.S. Hammers } \\
\text { J.S. Hammers } \\
\text { J.E. Andrews } \\
\text { J.S. Hammers } \\
\text { J.E. Andrews }\end{array}$ & 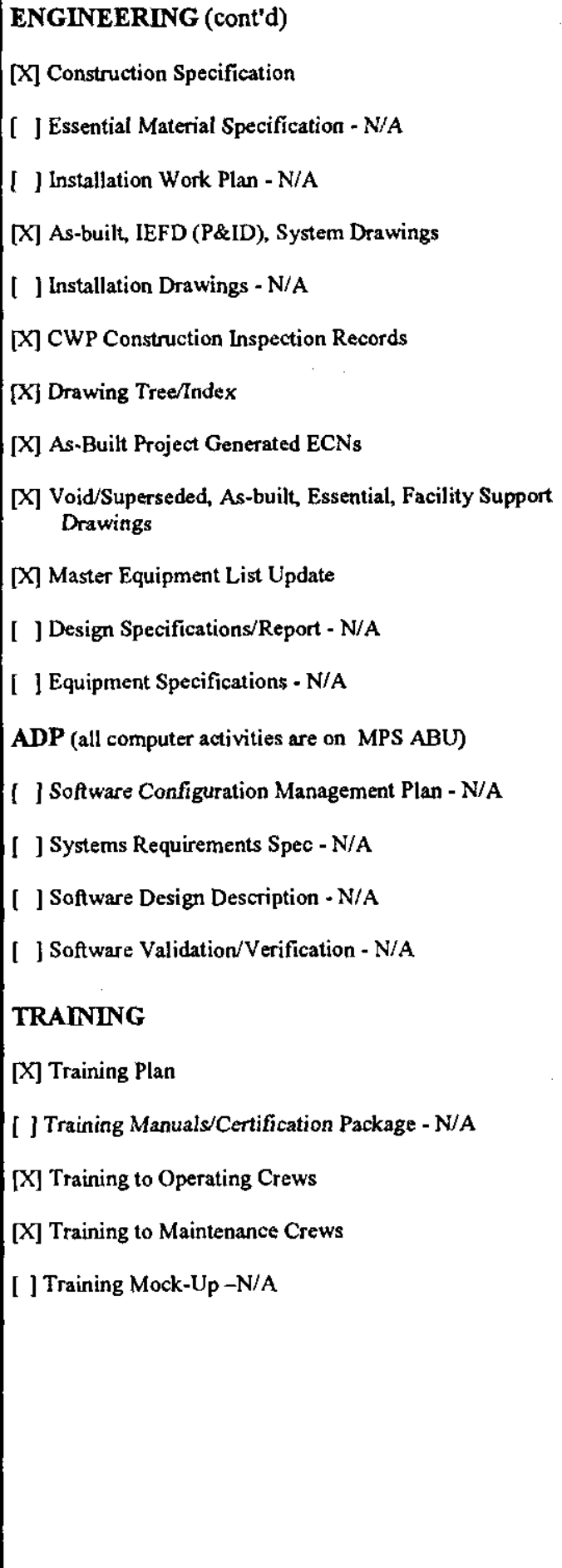 & $\begin{array}{l}\text { W.R. Swift } \\
\text { P.J. Bedell } \\
\text { P.J. Bedell } \\
\text { C.E. Shipler } \\
\text { C.E. Shipler }\end{array}$ \\
\hline
\end{tabular}


Program/Project Title: Project W-314, Tank Farm Restoration and Safe Operation, Phase 1 Component/System: AN-A Valve Pit Upgrades June 28, 1999

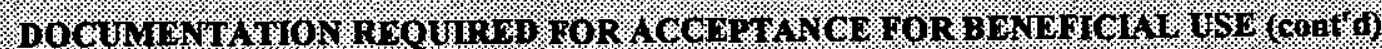

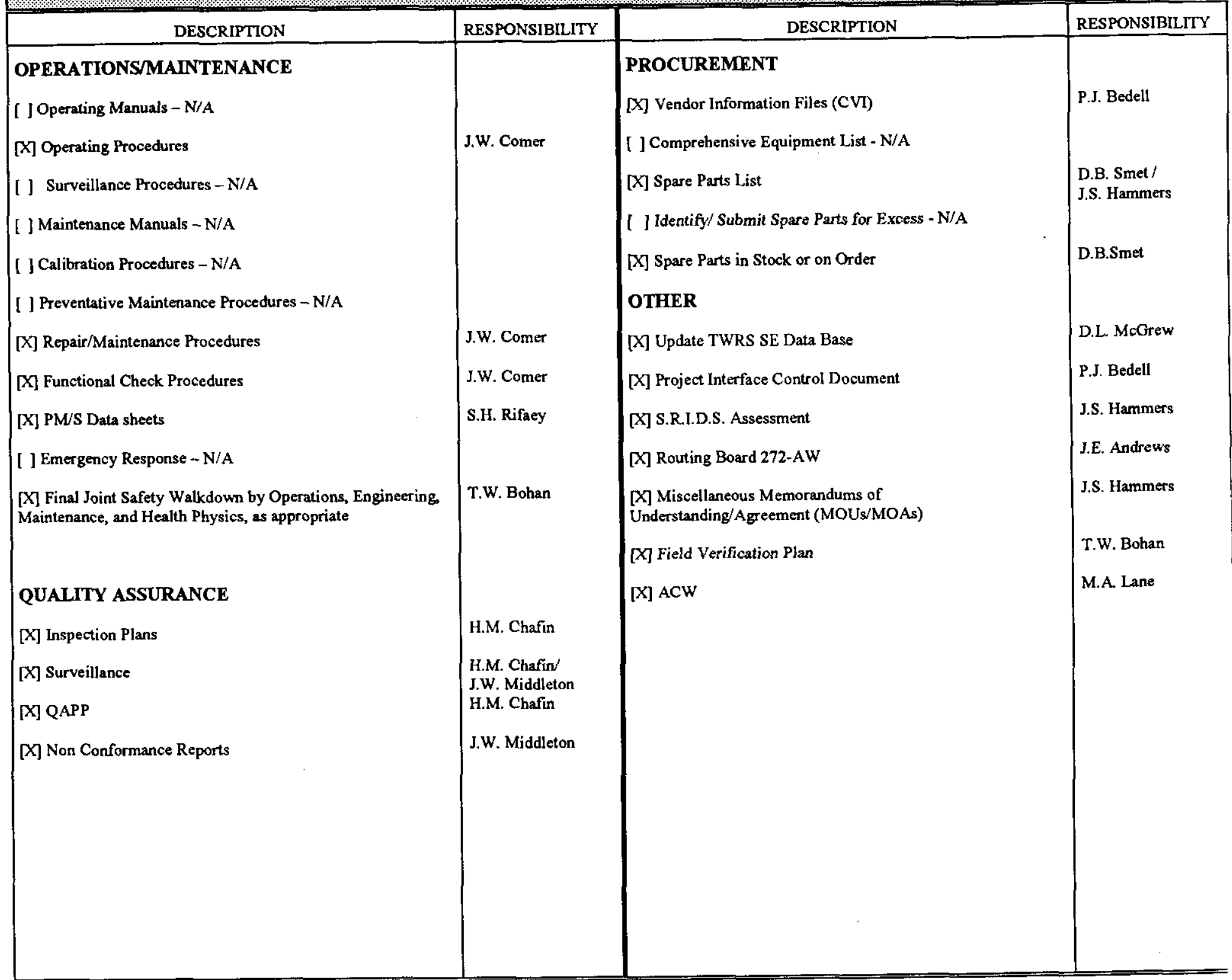




\section{ABU INDEX - Roadmap of Supporting Information}

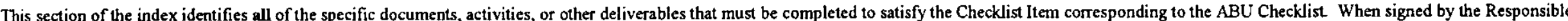
Persor it indicates completion and incorporation of W-314 requirements in the specific item. Operations Acceptance indicates completion of the Checklist Item for the specific facility.

\begin{tabular}{|c|c|c|c|c|}
\hline $\begin{array}{l}\text { CHECKLIST ITEM } \\
\text { (from ABU Chocklist) }\end{array}$ & $\begin{array}{l}\text { SUPPORTING DOCUMENT } \\
\text { NUMBER and TITLE } \\
\text { (or activity and its acceptance, or other } \\
\text { definitive information) }\end{array}$ & $\begin{array}{l}\text { RESPONSIBLE } \\
\text { PERSON } \\
\text { (by name \& org) }\end{array}$ & $\begin{array}{c}\text { OPERATIONS } \\
\text { ACCEPTANCE } \\
\text { (for specific, discreet portions) } \\
\text { When all items in block are } \\
\text { complete, the cover checklist } \\
\text { can be signed off }\end{array}$ & COMMENTS \\
\hline USQ Screening & $\begin{array}{l}\text { TF-97-0558, Rev 1, New Cover Block Design } \\
\text { for the 241-AN-A and 241-AN-B Valve Pits Per } \\
\text { W-314 } \\
\text { TF-97-0560, Rev 7, Nozzle Additions at 241- } \\
\text { AN-A and 241-AN-B Valve Pits } \\
\text { TF-97-0800, Rev 8, Project W-314 Valve Pit } \\
\text { Manifold Design for 241-AN-A and 241-AN-B } \\
\text { Valve Pit } \\
\text { TF-97-0835, Rev 3, Special Protective Coating } \\
\text { for Valve Pits Upgrade by Project W-314 } \\
\text { TF-97-0866, Rev 0, W314 Leak Detection } \\
\text { System Design for the 241-AN-A and 241-AN- } \\
\text { B Valve Pits } \\
\text { TF-98-0020, Rev 0, ECN 646103 New Gasket } \\
\text { Material for Purex Connector per W-314 } \\
\text { TF-98-0183, Rev 0, Electrical Power Supply for } \\
\text { Leak Detection Relay Cabinets AN } \\
\text { TF-98-0214, Rev 0, W314 Change Facility } \\
\text { Installation and Gate Modification } \\
\text { TF-98-0215, Rev 3, W314 Procurement } \\
\text { Specification for Impact Wrench AN Valve Pit } \\
\text { Upgrade } \\
\text { TF-98-0217, Rev 10, W314 Electrical Design } \\
\text { for Valve Pit Upgrade 241-AN-A,B } \\
\text { TF-98-0219, Rev 6, Modifications of W-314 } \\
\text { AN Valve Pit Jumper Drawings Due to Vendor } \\
\text { Information from Valve Procurement } \\
\text { TF-98-0229, Rev 21, ECN to Structural } \\
\text { Drawings for 241-AN-A \& 241-AN-B Valve Pit } \\
\text { TF-98-0237, Rev 1, New Transfer Piping per } \\
\text { Project W-314 } \\
\text { TF-98-0238, Rev 0. ECN to Instrumentation } \\
\text { Drawings for 241-AN-A and 241-AN-B Valve }\end{array}$ & $\begin{array}{l}\text { D.F. Carlstrom, } \\
\text { SAR Eng. Services }\end{array}$ & & \\
\hline
\end{tabular}




\section{ABU INDEX - Rosdimap of Supporting Information}

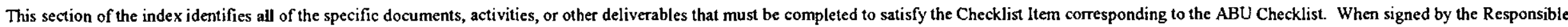
Person, it indicates completion and incorporation of W-314 requirements in the specific item. Operations Acceptance indicates completion of the Checklist Item for the specific facility.

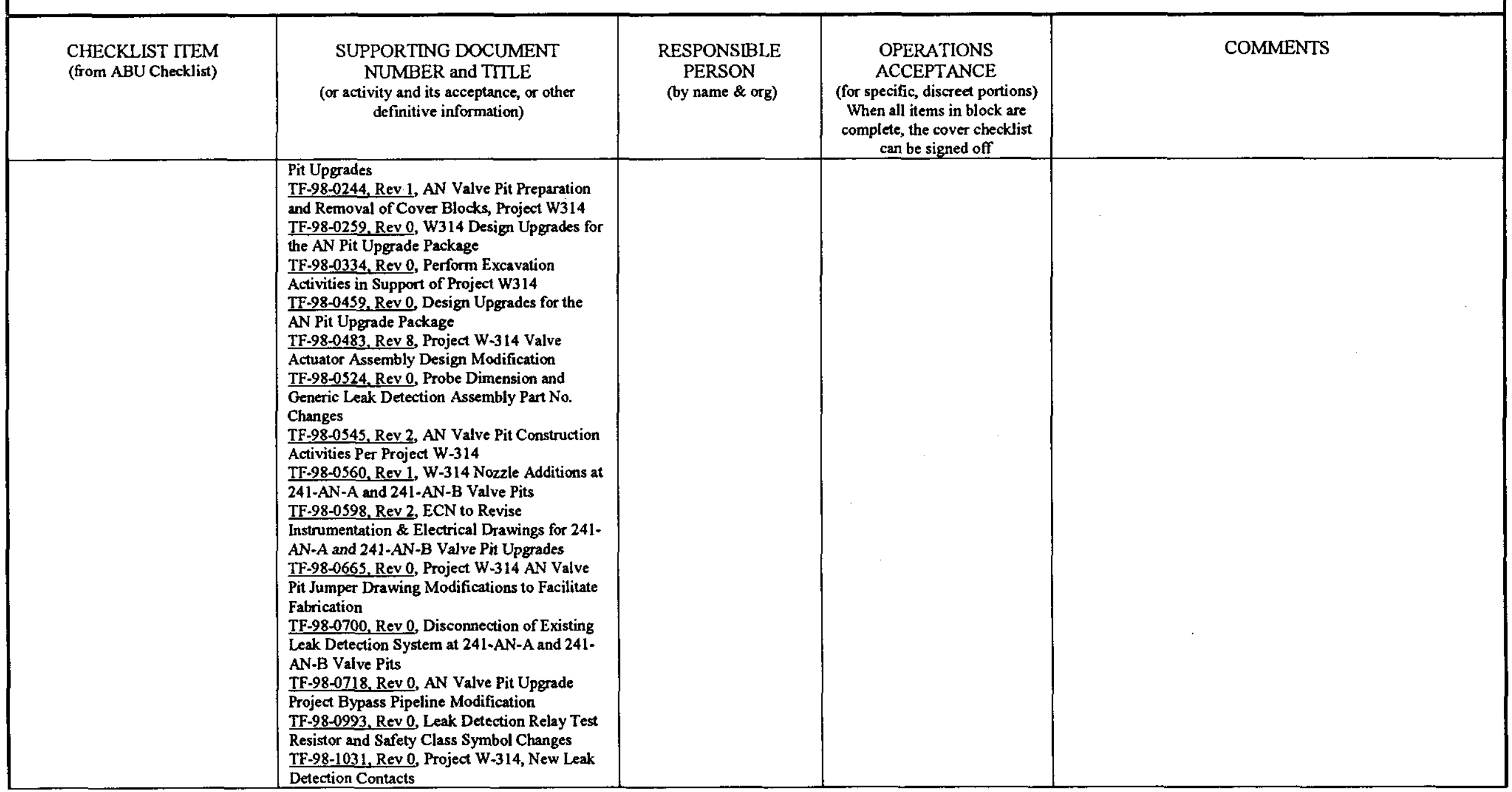


Program/Project Title: Project W-314, Tank Farm Restoration and Safe Operation, Phase 1

\section{ABU INDEX - Rosdinap of Supporting Tinformation}

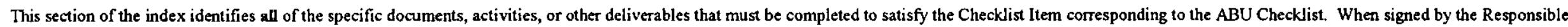
Person, it indicates completion and incorporation of W-314 requirements in the specific item. Operations Acceptance indicates completion of the Checklist Item for the specific facility.

\begin{tabular}{|c|c|c|c|c|}
\hline $\begin{array}{l}\text { CHECKLIST ITEM } \\
\text { (from ABU Checklist) }\end{array}$ & $\begin{array}{l}\text { SUPPORTING DOCUMENT } \\
\text { NUMBER and TITLE } \\
\text { (or activity and its acceptance, or other } \\
\text { definitive information) }\end{array}$ & $\begin{array}{l}\text { RESPONSIBLE } \\
\text { PERSON } \\
\text { (by name \& org) }\end{array}$ & $\begin{array}{c}\text { OPERATIONS } \\
\text { ACCEPTANCE } \\
\text { (for specific, discreet portions) } \\
\text { When all items in block are } \\
\text { complete, the cover checklist } \\
\text { can be signed off }\end{array}$ & COMMENTS \\
\hline . & $\begin{array}{l}\text { TF-98-1053, Rev 1, Valves Hydrotest and Seat } \\
\text { Leakage Tests Procedures Modifications } \\
\text { TF-98-1058, Rev 0, Leak Detection Relay Test } \\
\text { Resistor and Safety Class Symbol Changes } \\
\text { TF-98-1144, Rev 0, ECN W314-4C-041 Leak } \\
\text { Detection Power Supply Part Number Change } \\
\text { TF-98-1210, Rev 0, Modifications to Cover } \\
\text { Block Dimensions, Cathodic Protection and } \\
\text { Valve Position Sensor Wiring } \\
\text { TF-98-1212, Rev 0, Rebar Replacement and } \\
\text { Tolerance Changes to Construction } \\
\text { Specification W-314-C1 } \\
\text { TF-98-1219, Rev 1, Valve Position Sensor } \\
\text { Condulet Box Mounting Bracket } \\
\text { TF-98-1279, Rev 0, Leak Detection Relay } \\
\text { Cabinet NEMA 4 Alarms } \\
\text { TF-99-0121, Rev 0, ECN 314-4C-062 AN } \\
\text { Valve Pit Upgrade Instrument Terminal Box } \\
\text { Footing Concrete Change } \\
\text { TF-99-0128, Rev 0, ECN 314-4C-066 AN } \\
\text { Valve Pit Upgrade Leak Detection Panel } \\
\text { Breather/Drain Fitting Change } \\
\text { TF-99-0167, Rev 0, ECN 314-4C-076 AN } \\
\text { Valve Pit Upgrade Leak Detection Panel Tag } \\
\text { Number and Wiring Change } \\
\text { TF-99-0240, Rev 1, Miscellaneous Changes } \\
\text { Associated with Nozzle Installation at 241-AN. } \\
\text { A and 241-AN-B Valve Pits } \\
\text { TF-99-0248, Rev 0, ECN 314-4C-085 AN } \\
\text { Valve Pit Upgrade Leak Detection Panel } \\
\text { Selector Switch Contact Block Change } \\
\text { TF-99-0422, Rev 0, ECN 314-4C-105 AN } \\
\text { Valve Pit Upgrade Leak Detection Element }\end{array}$ & & $x_{2}$ & \\
\hline
\end{tabular}


$0 Z$ jo 8 ว8̊

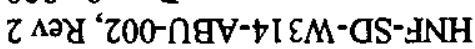

\begin{tabular}{|c|c|c|c|c|}
\hline & & 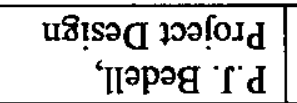 & 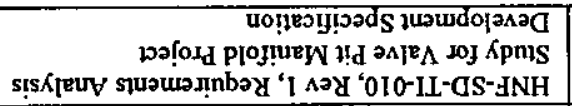 & 1dəวuoว usి!səa \\
\hline & & 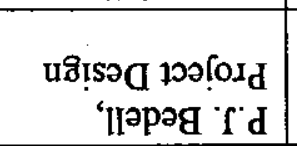 & 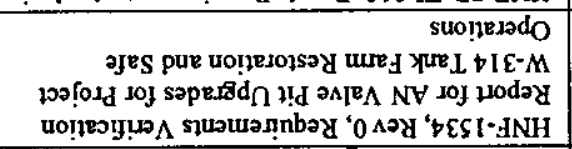 & 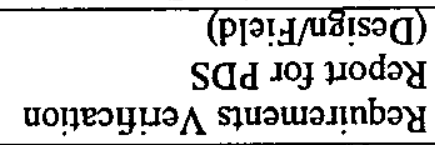 \\
\hline & & 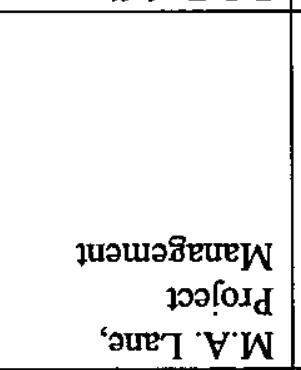 & 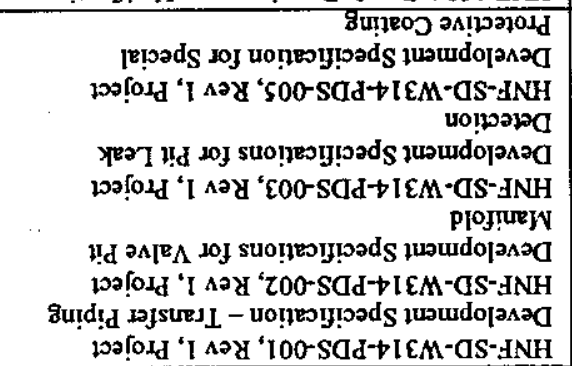 & 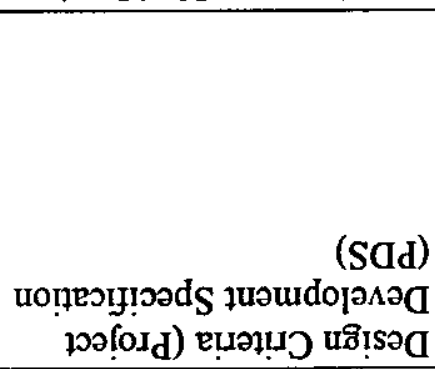 \\
\hline & & 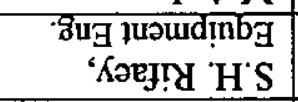 & I $A \partial \mathrm{y}$ '000-Tas-WM-as'-INH & 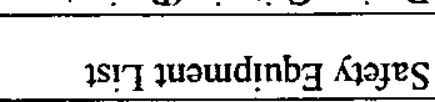 \\
\hline & & & 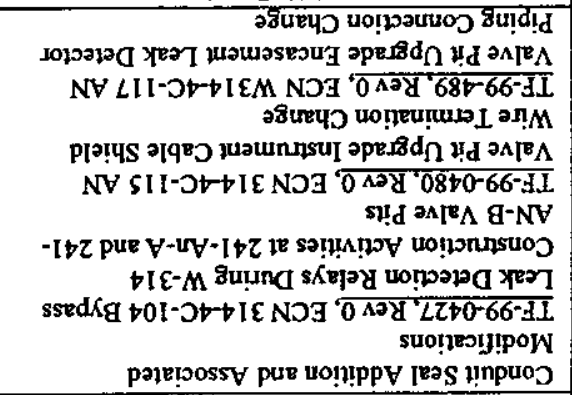 & 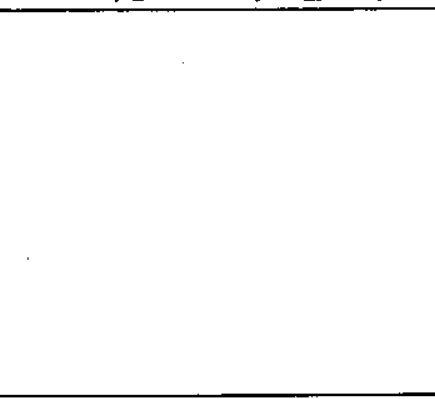 \\
\hline SLNGWWOD & 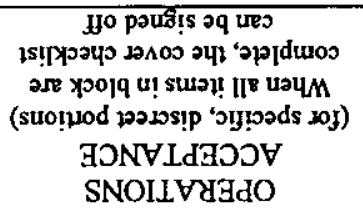 & 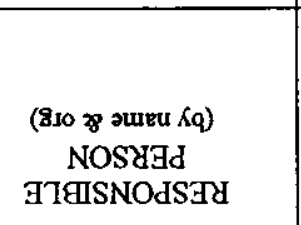 & 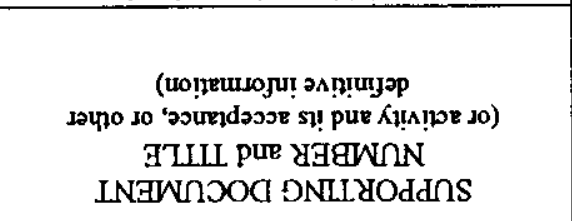 & 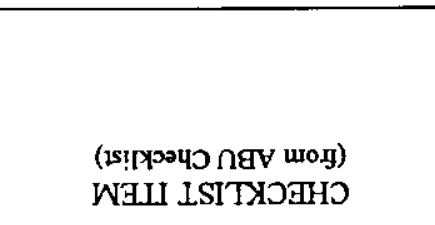 \\
\hline \multicolumn{5}{|c|}{ 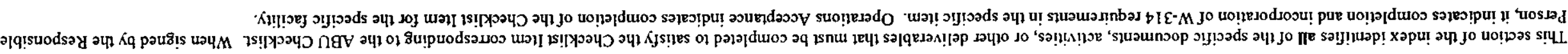 } \\
\hline \multicolumn{5}{|c|}{ 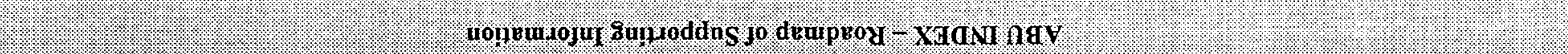 } \\
\hline
\end{tabular}




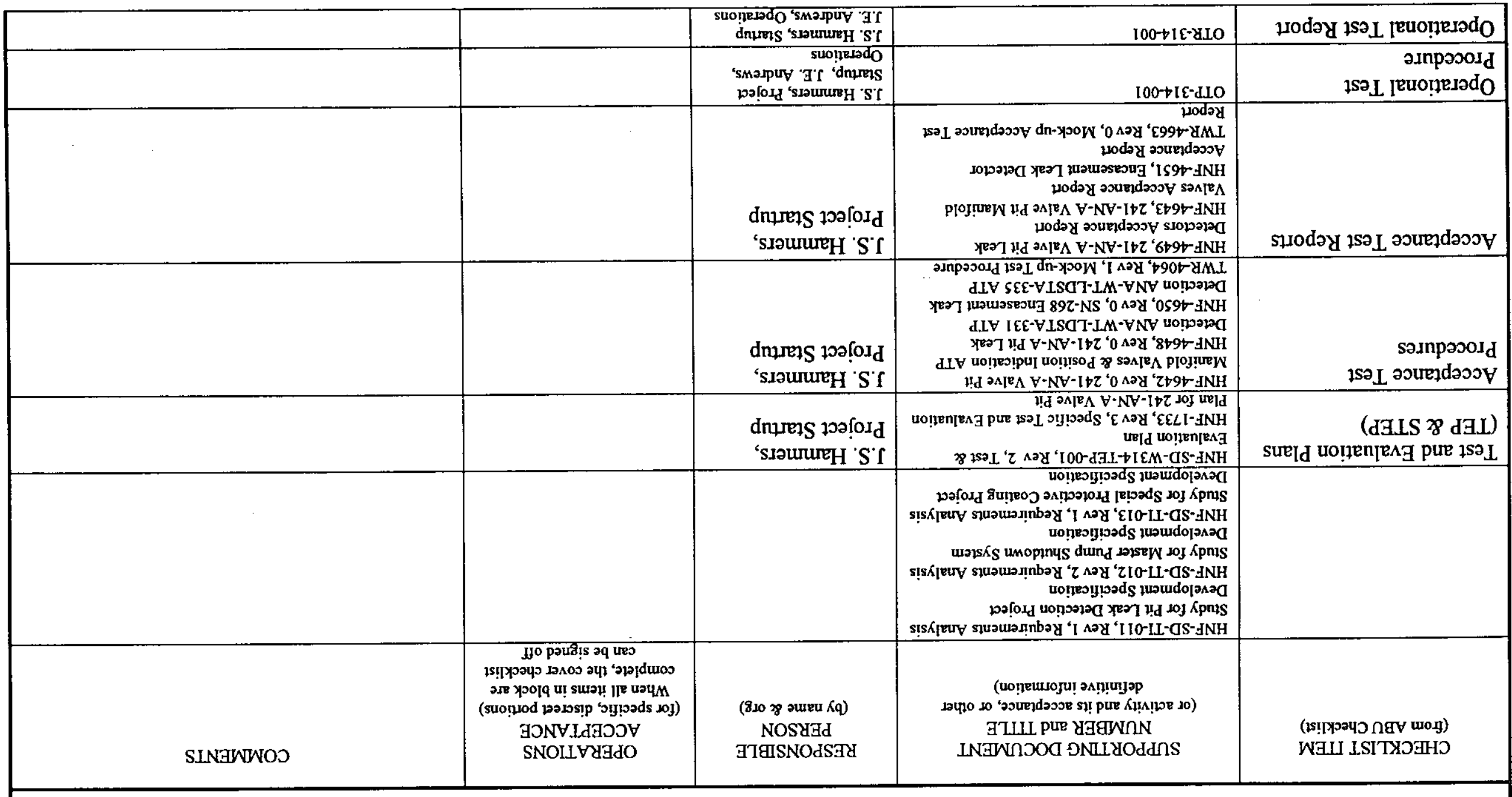

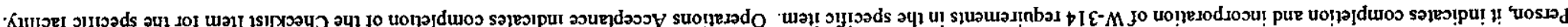

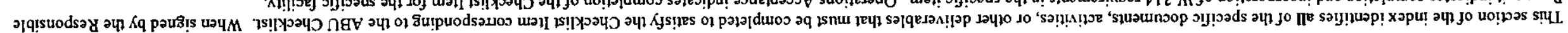

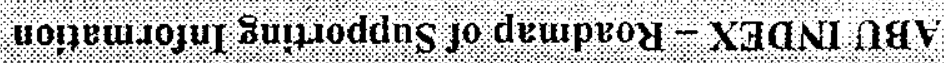

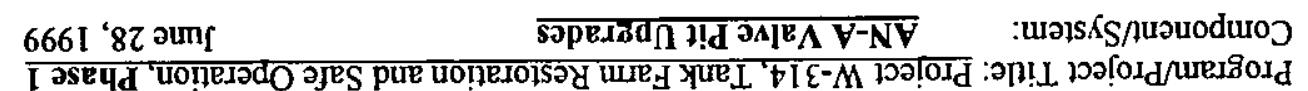




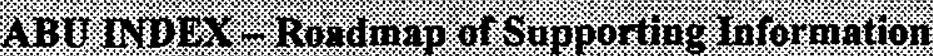

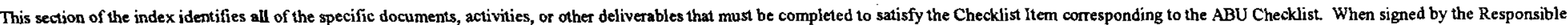
Person, it indicates completion and incorporation of W-314 requirements in the specific item. Operations Acceptance indicates completion of the Checklist Item for the specific facility.

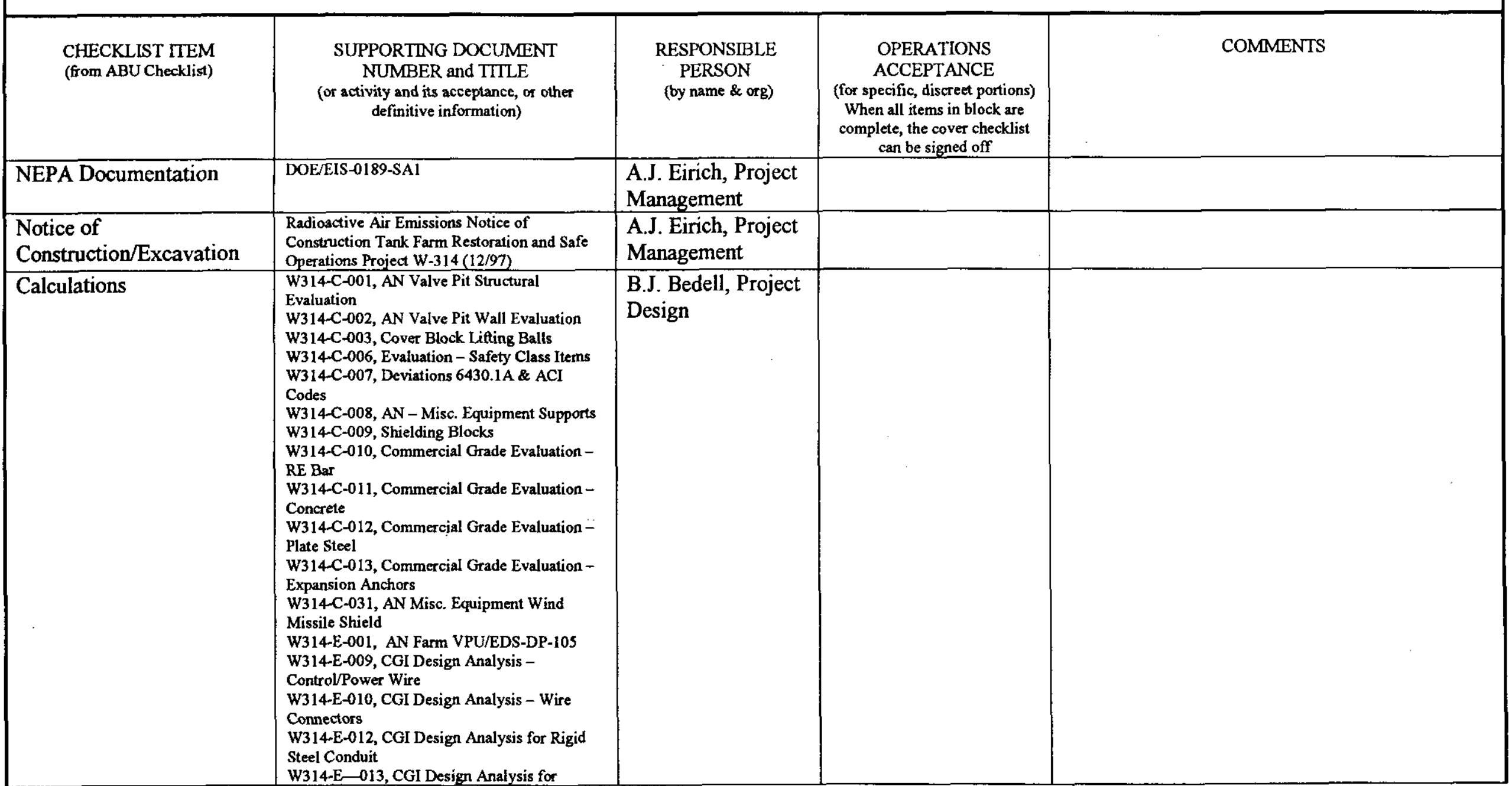

HNF-SD-W314-ABU-002, Rev 2

Page 10 of 20 
Program/Project Title: Project W-314. Tank Farm Restoration and Safe Operation, Phase 1

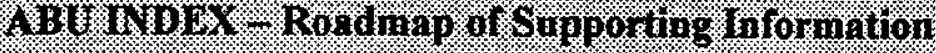

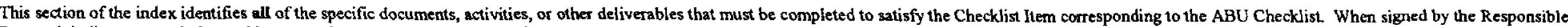
Person, it indicates completion and incorporation of W-314 requirements in the specific item. Operations Acceptance indicates completion of the Checklist Item for the specific facility.

\begin{tabular}{|c|c|c|c|c|}
\hline $\begin{array}{l}\text { CHECKLIST ITEM } \\
\text { (from ABU Checklist) }\end{array}$ & $\begin{array}{l}\text { SUPPORTING DOCUMENT } \\
\text { NUMBER and TITLE } \\
\text { (or activity and its acceptance, or other } \\
\text { definitive information) }\end{array}$ & $\begin{array}{l}\text { RESPONSIBLE } \\
\text { PERSON } \\
\text { (by name \& org) }\end{array}$ & $\begin{array}{c}\text { OPERATIONS } \\
\text { ACCEPTANCE } \\
\text { (for specific, discreet portions) } \\
\text { When all items in block are } \\
\text { complete, the cover checklist } \\
\text { can be signed off }\end{array}$ & COMMENTS \\
\hline & $\begin{array}{l}\text { Conduit Fittings/ Outlet Boxes } \\
\text { W314-E-014, CGI Design Analysis for Molded } \\
\text { Case Circuit Breakers } \\
\text { W314-E-021, Cathodic Protection Analysis } \\
\text { 241-AN-A \& 241-AN-B Pit Mods } \\
\text { W314-I-001, Ammount of Liquid/Waste Present } \\
\text { in the bottom of AN Valve Pit at time of } \\
\text { detection } \\
\text { W314-I-002, Human Factors Analysis Pit Leak } \\
\text { Detection Warning Lights } \\
\text { W314I-003, Pit Leak Detection System } \\
\text { Refiability/Probability of Failures } \\
\text { w314-I-004, Solar Effects on Relay Cabinet } \\
\text { Enclosure } \\
\text { W314-I-005, Solar Effects on Valve Position } \\
\text { Sensor Enclosure } \\
\text { W314-I-006, Safety Class Instrumentation } \\
\text { Analysis } \\
\text { W314i-1007, Commercial Grade MTL 2313 } \\
\text { Trip Amptifier Dedicated to Safety Class } \\
\text { W314-I-008, Commercial Grade B/W Leak } \\
\text { Detection Relay Dedicated to Safety Class } \\
\text { W314-I-009, Commercial Grade Stainless Steel } \\
\text { Probes Dedicated to safety } \\
\text { W314-I-010, Commercial Grade Warning Light } \\
\text { Transformer Dedicated to Safety Class } \\
\text { W314-I-011, Commercial Grade Edwards } \\
\text { Strobe Local Warning Light Dedicated to Safety } \\
\text { Class } \\
\text { W314-1-012, Commercial Grade Potter \& } \\
\text { Brumfieid Latch Relay Dedicated to Safety } \\
\text { Class } \\
\text { W314-I-013, Commercial Grade Potter \& }\end{array}$ & & & \\
\hline
\end{tabular}

HNF-SD-W314-ABU-002, Rev 2

Page 11 of 20 







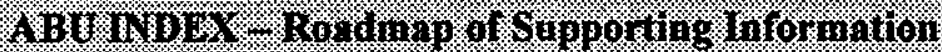

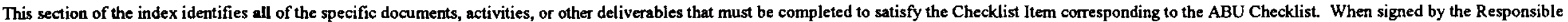
Person, it indicates completion and incorporation of W-314 requirements in the specific item. Operations Acceptance indicates completion of the Checklist ltem for the specific facility.

\begin{tabular}{|c|c|c|c|c|}
\hline $\begin{array}{l}\text { CHECKLIST ITEM } \\
\text { (from ABU Checklist) }\end{array}$ & $\begin{array}{l}\text { SUPPORTING DOCUMENT } \\
\text { NUMBER and TITLE } \\
\text { (or activity and its acceptance, or other } \\
\text { definitive information) }\end{array}$ & $\begin{array}{l}\text { RESPONSIBLE } \\
\text { PERSON } \\
\text { (by name \& org) }\end{array}$ & $\begin{array}{c}\text { OPERATIONS } \\
\text { ACCEPTANCE } \\
\text { (for specific, discreet portions) } \\
\text { When all items in block are } \\
\text { complete, the cover checklist } \\
\text { can be signed off }\end{array}$ & COMMENTS \\
\hline & $\begin{array}{l}\text { W314-P-017, } 6 \text {-in. Encasement Closure Design } \\
\text { in accordance with ASME B31.3 } \\
\text { W314-P-018, 4-in. Encasement Closure Design } \\
\text { per ASME B31.3 } \\
\text { W314-P-019, Failure Modes and Effects } \\
\text { Analysis for Project W-314 } \\
\text { W314-P-020, Engineering Evaluation of } \\
\text { Commercial Grade Items } \\
\text { W314-P-021, Stresses Induced on Piping Due to } \\
\text { Loads on Integral Attachments Class } \\
\text { W314-P-022, Shielding Block of Handle } \\
\text { Extension of Valve } \\
\text { W314-P-032, Funnel Collar Pin Analysis } \\
\text { W314-P-038, Valve Nozzle Loads Analysis for } \\
\text { Valve Manifolds in Vaive Pits 241-AN-A\&B } \\
\text { W314-P-051, Pressure Design of 14" ENC. For } \\
\text { WT-V-110 } \\
\text { W314-P-053, Pressure Design for Transfer } \\
\text { Piping and Encasements } \\
\text { W314-P-054, CGI Evaluation Encasement } \\
\text { Valves } \\
\text { W314-P-055, Valve Pit Ambient Temperature }\end{array}$ & & & \\
\hline $\begin{array}{l}\text { Commercial Grade Item } \\
\text { Dedication Documentation }\end{array}$ & $\begin{array}{l}\text { W-314-C1-2, Reinforcing Steel CGI Data } \\
\text { W-314-C1-6, Chemical Resistant } \\
\text { Decontamanatable Coatings, List of Materials } \\
\text { W-314-C1-7, Chemical Resistant } \\
\text { Decontamanatable Coatings, Installers } \\
\text { Certificate } \\
\text { W-314-C1-20, Chemical Process Piping } \\
\text { Systems, Certified Material Test Reports } \\
\text { W-314-C1-20.1, Chemical Process Piping } \\
\text { Systems Weld Filler, Certified Material Test } \\
\text { Reports }\end{array}$ & $\begin{array}{l}\text { P.J. Bedell, Project } \\
\text { Design }\end{array}$ & & \\
\hline
\end{tabular}

HNF-SD-W314-ABU-002, Rev 2

Page 13 of 20 
Program/Project Title: Project W-314, Tank Farm Restoration and Safe Operation, Phase 1

Component/System: AN-A Valve Pit Upgrades June 28, 1999

\section{ABU INOEX - Rondinap or Supporting Intormation}

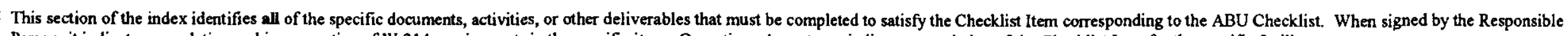
Person, it indicates completion and incorporation of W-314 requirements in the specific item. Operations Acceptance indicates completion of the Checklist Item for the specific facility.

\begin{tabular}{|c|c|c|c|c|}
\hline $\begin{array}{l}\text { CHECKLIST ITEM } \\
\text { (from ABU Checklist) }\end{array}$ & $\begin{array}{l}\text { SUPPORTING DOCUMENT } \\
\text { NUMBER and TITLE } \\
\text { (or activity and its acceptance, or other } \\
\text { definitive information) }\end{array}$ & $\begin{array}{c}\text { RESPONSIBLE } \\
\text { PERSON } \\
\text { (by name \& org) }\end{array}$ & $\begin{array}{c}\text { OPERATIONS } \\
\text { ACCEPTANCE } \\
\text { (for specific, discreet portions) } \\
\text { When all items in block are } \\
\text { complete, the cover checklist } \\
\text { can be signed off }\end{array}$ & COMMENTS \\
\hline & $\begin{array}{l}\text { W-314-C1-20.2, Chemical Process Piping } \\
\text { Systems Weld Filler, Certified Material Test } \\
\text { Reports } \\
\text { W-314-C1-20.3, Chemical Process Piping } \\
\text { Systems Weld Filler, Certified Material Test } \\
\text { Reports } \\
\text { W-314-C1-21, Pipe Code M-26a, CGI Data } \\
\text { W-314-Cl-22, Fittings Pipe Code M-26a CGI } \\
\text { Data PO 501-5.GPBF-02 } \\
\text { W-314-C1-22.1, CGI Data for Buttwelded } \\
\text { Fittings M26a, PO 501-5-GPJN-01 } \\
\text { W-314-C1-22.2, CGI Data for Buttwelded } \\
\text { Fittings M26a, PO 501-5-GPJN-02 } \\
\text { W-314-C1-30, Intrinsically Safe Control Relay } \\
\text { W-314-C1-31, Trip Amplifier } \\
\text { W-314-C1-32, Strobe Light } \\
\text { W-314-C1-33, General Purpose Relay } \\
\text { W-314-C1-34, Latching Reiay } \\
\text { W-314-C1-35, Transformer } \\
\text { W-314-C1-36, Power Supply } \\
\text { W-314-C1-37, Heater } \\
\text { W-314-C1-38, Terminal Block } \\
\text { W-314-C1-39, Disconnect Switch } \\
\text { W-314-C1-40, Intrinsically Safe Control Relay } \\
\text { W-314-Cl-41, Trip Amplifier } \\
\text { W-314-C1-42, Dual Coil Latch Relay } \\
\text { W-314-CI-43, General Purpose Relay } \\
\text { W-314-C1-44, Intrinsically Safe Switch } \\
\text { Operated Relay } \\
\text { W-314-C1-45, Transformer } \\
\text { W-314-C1-46, Power Supply } \\
\text { W-314-C1-47, Heater } \\
\text { W-314-C1-48, Terminal Block } \\
\end{array}$ & & & . \\
\hline
\end{tabular}


$0 Z$ Jo $\varsigma$ ว $288 \mathrm{e}_{\mathbf{d}}$

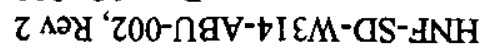

\begin{tabular}{|c|c|c|c|c|}
\hline & & 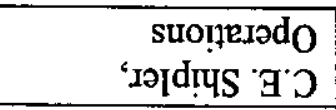 & 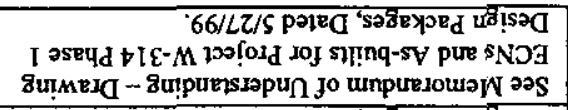 & $\begin{array}{r}\text { Joddns ' [e!̣urss } \\
\text { 'H!nq-sY 'popasıadn } S / p ! \circ \Lambda \\
\end{array}$ \\
\hline & & 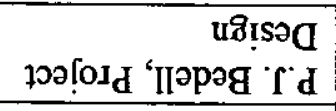 & 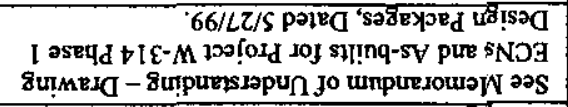 & 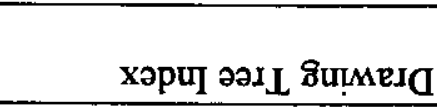 \\
\hline & & 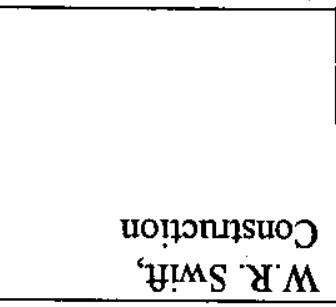 & 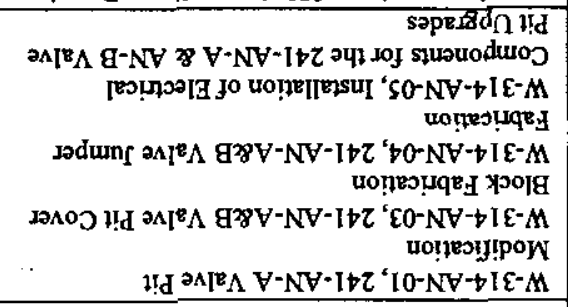 & 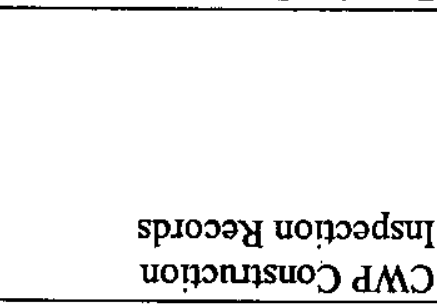 \\
\hline & & 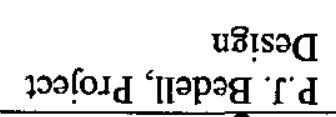 & 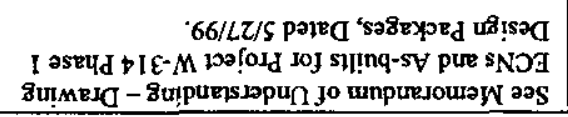 & 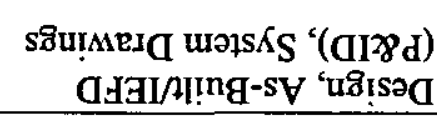 \\
\hline & & 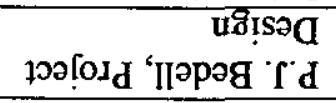 & 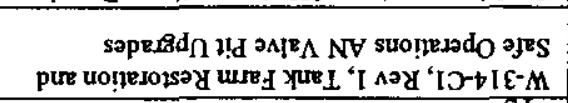 & uo!̣eoly!oods uononusuo? \\
\hline & & 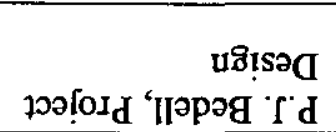 & 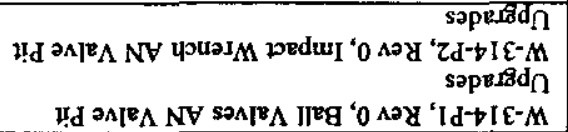 & 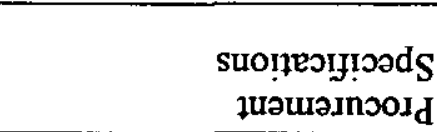 \\
\hline & & & 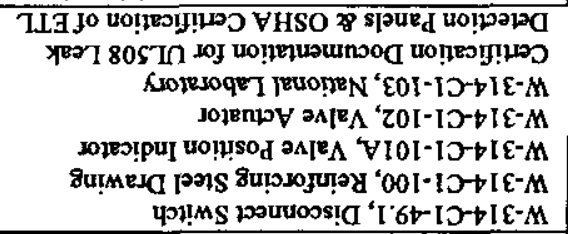 & \\
\hline SINTIWWOD & 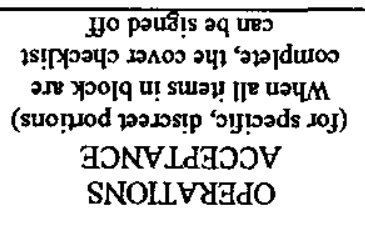 & $\begin{array}{l}\text { (810 дg эuru Kq) } \\
\text { NOS\&Ad } \\
\text { 37gISNOdSGy }\end{array}$ & 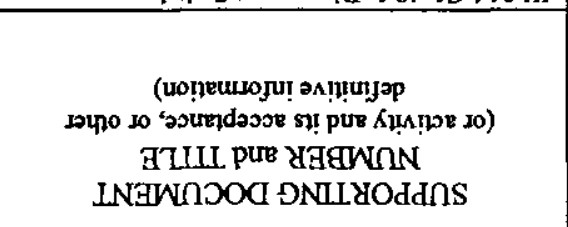 & 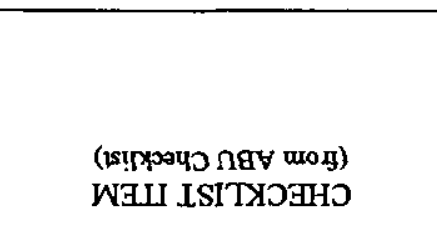 \\
\hline \multicolumn{5}{|c|}{ 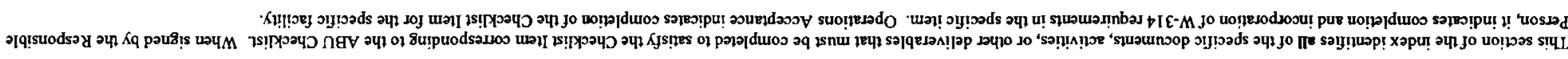 } \\
\hline \multicolumn{5}{|c|}{ 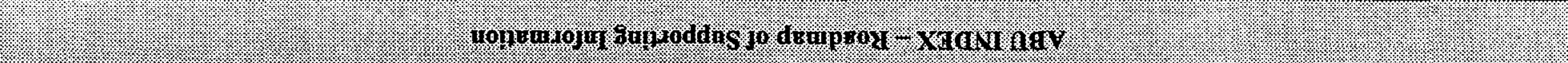 } \\
\hline
\end{tabular}

\author{
666I 'sZ aun

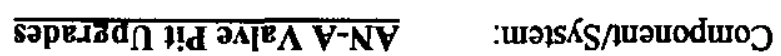

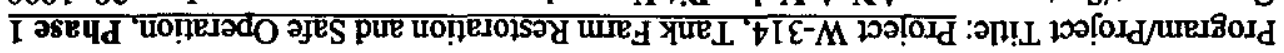




\begin{tabular}{|c|c|c|c|c|}
\hline & & 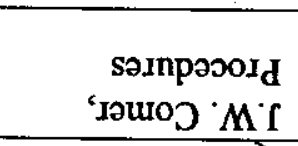 & 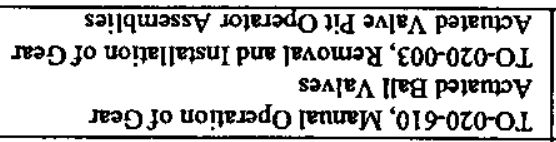 & 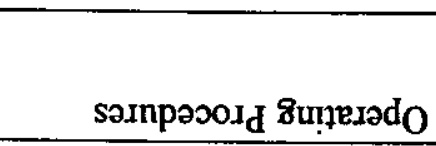 \\
\hline & & $\begin{array}{r}\forall \mathrm{O} \\
\text { 'uoləpp!W } \mathrm{M} \cdot \mathrm{C}\end{array}$ & & şodəy asureuuojuo \\
\hline & & VO 'ugечD'W'H & $Z \Lambda \partial \gamma$ ' $100-d d \forall O+I E M-a S^{-}-3 N H$ & ddVठ \\
\hline & & 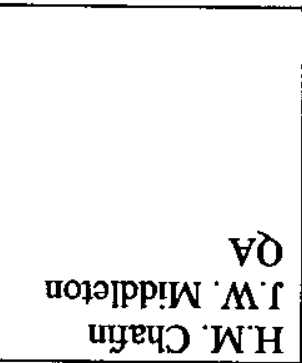 & 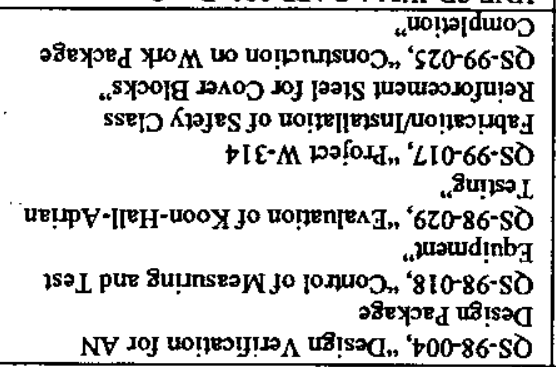 & 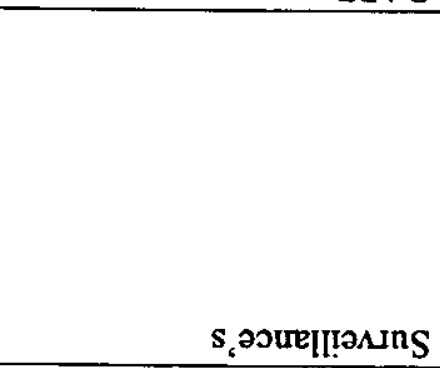 \\
\hline & & uyeนว $\mathrm{WH}$ & & sur [d uọpordsuI \\
\hline & & $\begin{array}{r}\text { 8utuleII } \\
\text { 'swow 'W' }\end{array}$ & & 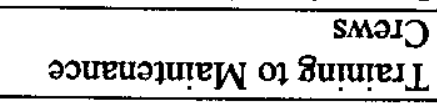 \\
\hline & & $\begin{array}{r}\text { gu!u!e.I } \\
\text { stwoW 'W' } \\
\end{array}$ & & 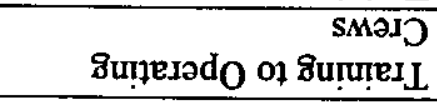 \\
\hline & & $\begin{array}{r}\text { 8u!u!teII } \\
\text { 'suow WI } \\
\end{array}$ & $+00-86-0096 L$ & 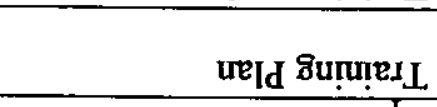 \\
\hline & & 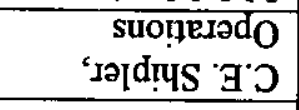 & & 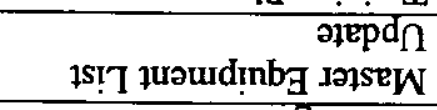 \\
\hline & & & & SôUMMEId \\
\hline SINGWWOO & 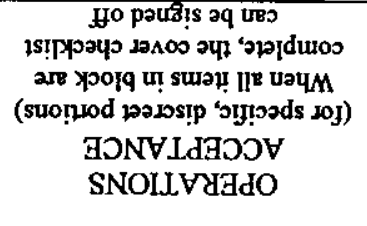 & 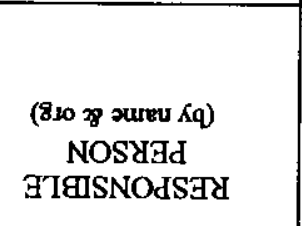 & 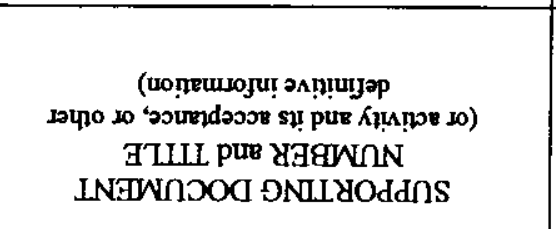 & 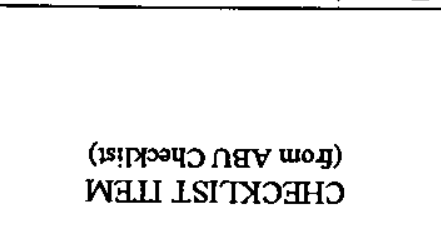 \\
\hline \multicolumn{5}{|c|}{ 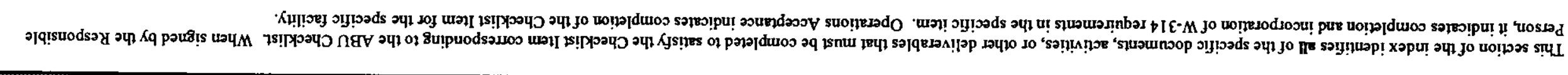 } \\
\hline \multicolumn{5}{|c|}{ 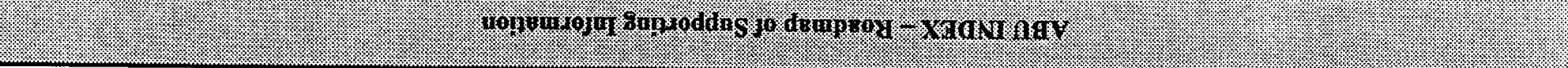 } \\
\hline
\end{tabular}




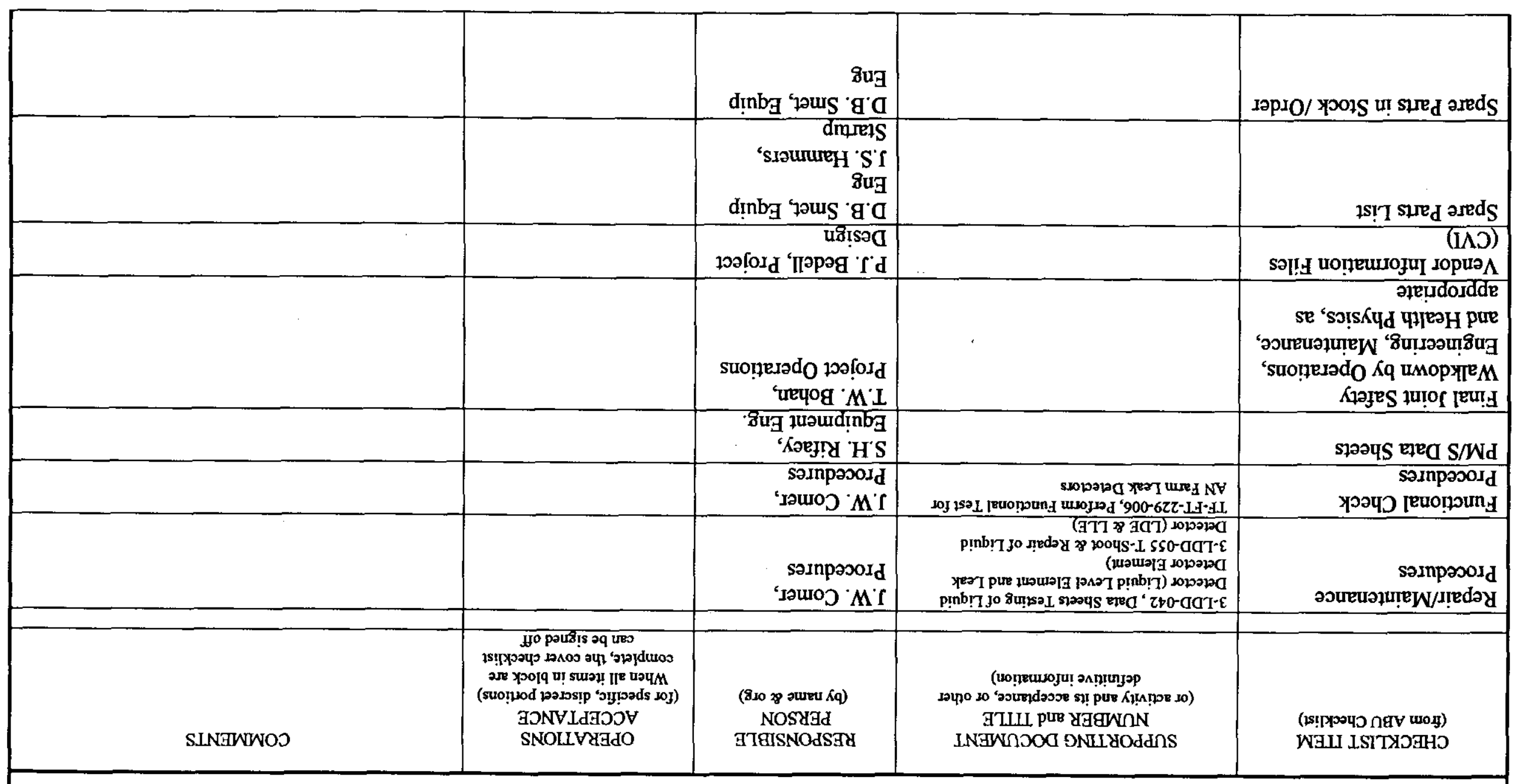

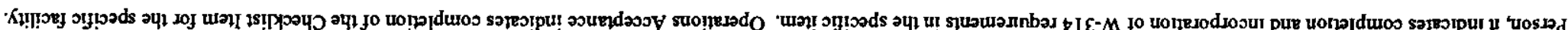

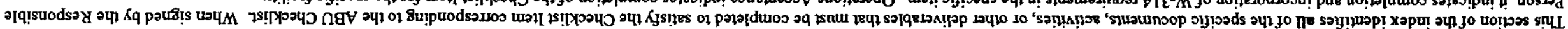

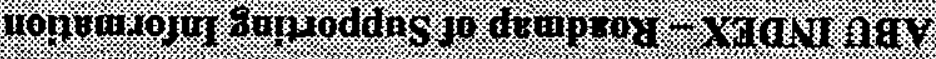




\begin{tabular}{|c|c|c|c|c|}
\hline & & 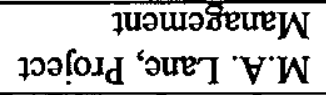 & & MOV \\
\hline & & 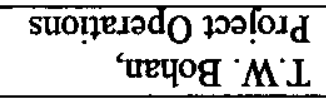 & $58 \varepsilon t-Y M I$ & 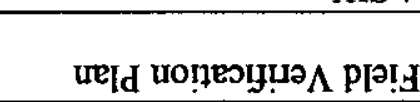 \\
\hline & & $\begin{array}{r}\text { dnure's } \\
\text { 'slaurureH 'S' } \\
\end{array}$ & 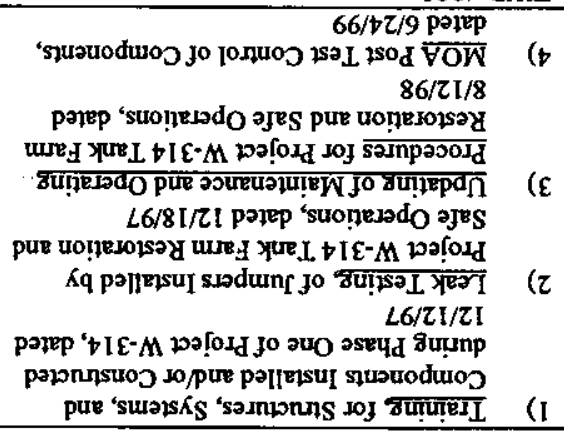 & 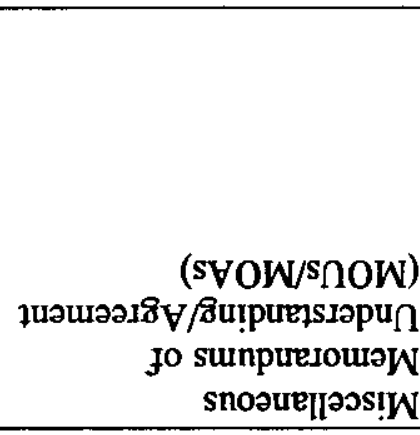 \\
\hline & & $\begin{array}{r}\text { suoṇerado } \\
\text { sMarpur } \cdot \mathrm{g} \cdot \mathrm{C}\end{array}$ & 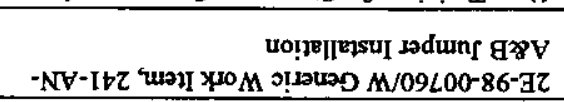 & $M \forall Z L Z$ - preog su!̣noy \\
\hline & . & 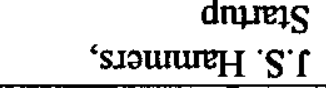 & 0 AวY " $669 \varepsilon-\mathrm{E}$ - & 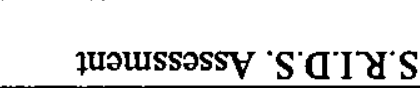 \\
\hline & & 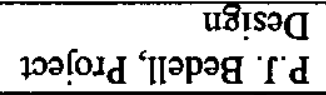 & & 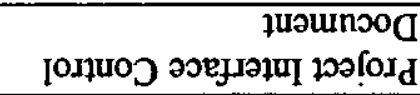 \\
\hline & & sKS 'MOIDOW ' $\mathrm{T}$ ' & & eled as SyML ә̇epd $\Lambda$ \\
\hline S.INGINWOO & 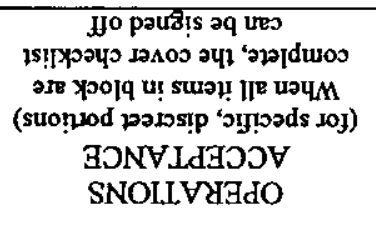 & $\begin{array}{l}\text { (810 \% गureu Kq) } \\
\text { NOS4Gd } \\
\text { GTGISNOdS }\end{array}$ & 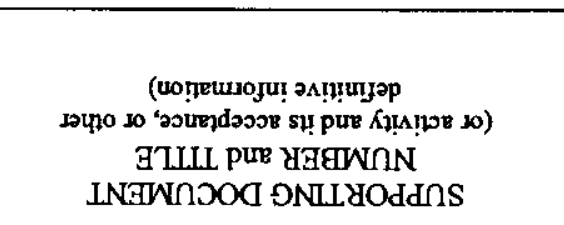 & 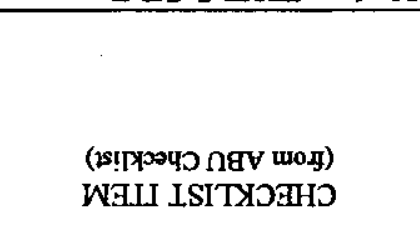 \\
\hline \multicolumn{5}{|c|}{ 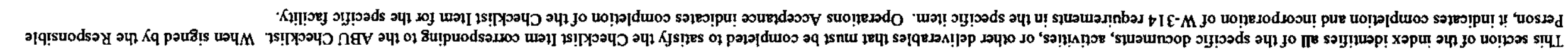 } \\
\hline \multicolumn{5}{|c|}{ 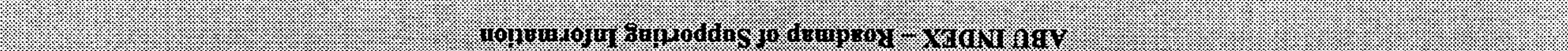 } \\
\hline
\end{tabular}


Program/Project Title: Project W-314. Tank Farm Restoration and Safe Operation. Phase 1

Component/System: AN-A Valve Pit Upgrades

June 28, 1999

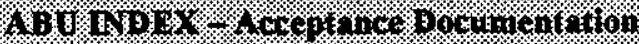

FINAL ACCEPTANCE SIGNATURES

ABU-002 for AN-A Valve Pit Upgrades
All deliverables under "Supporting Document Number and Title above are complete, signed, and acceptable or are included on an approved punchlist. Tank Farm Operations accepts full responsibility for systems, structures, and components covered by this ABU.
Facility Manager

Date

W-314 Operations Manager

Date

Design Authority

Date

TWRS Chief Engineer

Date 


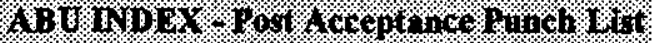

This section of the index identifies items still requiring completion or closure after acceptance of the segment/system per the ABU. Note: Items affecting Readiness may not be on the punch list without documented justification, an approved plan to correct the deficiency, and written concurrence from the Director of Tank Farms (or his delegee).

\begin{tabular}{|c|c|c|c|}
\hline ITEM & $\begin{array}{c}\text { RESPONSIBLE PERSON } \\
\text { (Signature) } \\
\text { AND ORGANIZATION }\end{array}$ & $\begin{array}{l}\text { COMMTTMENT } \\
\text { DATE }\end{array}$ & ACCEPTANCE OF COMPLETION \\
\hline & & & \\
\hline & & & \\
\hline & & & \\
\hline & & & \\
\hline & & & \\
\hline & & & \\
\hline & & & \\
\hline & & & \\
\hline & & & \\
\hline & & & \\
\hline & & & \\
\hline & & & \\
\hline & & & \\
\hline End of Table & & & \\
\hline
\end{tabular}




\section{DISTRIBUTION SHEET}

\begin{tabular}{|c|c|c|c|c|c|}
\hline \multirow{2}{*}{$\begin{array}{l}\text { TO } \\
\text { DISTRIBUTION }\end{array}$} & \multirow{2}{*}{\multicolumn{3}{|c|}{$\begin{array}{l}\text { From } \\
\text { Tank Farm Restoration and Safe } \\
\text { Operation }\end{array}$}} & \multicolumn{2}{|l|}{ Page 1 of 1} \\
\hline & & & & \multicolumn{2}{|l|}{ Date $6 / 7 / 99$} \\
\hline \multicolumn{4}{|l|}{ Project Title/Work Order } & \multicolumn{2}{|l|}{ EDT No. N/A } \\
\hline \multicolumn{4}{|c|}{$\begin{array}{l}\text { Project W-314 /HNF-SD-W314-ABU-002 Rev. 2, } \\
\text { HNF-SD-W314-ABU-003, Rev. } 2\end{array}$} & \multicolumn{2}{|c|}{ ECN No. 654217} \\
\hline Name & MSIN & $\begin{array}{l}\text { Text } \\
\text { With All } \\
\text { Attach. }\end{array}$ & Text Only & $\begin{array}{l}\text { Attach./ } \\
\text { Appendix } \\
\text { Only }\end{array}$ & $\begin{array}{l}\text { EDT/ECN } \\
\text { Only }\end{array}$ \\
\hline $\begin{array}{l}\text { T. W. Bohan } \\
\text { D. E. Bowers } \\
\text { W. E. Bryan } \\
\text { H. M. Chafin } \\
\text { J. W. Comer } \\
\text { R. A. Dodd } \\
\text { J. D. Galbraith } \\
\text { J. S. Gilbert } \\
\text { J. S. Hammers } \\
\text { J. R. LaPointe } \\
\text { J. W. Lentsch } \\
\text { D. L. McGrew } \\
\text { J. M. Morris } \\
\text { S. H. Rifaey } \\
\text { C. E. Shipler } \\
\text { B. L. Syverson } \\
\text { M. W. Tiffany } \\
\text { Project Files } \\
\text { Construction Doc Control }\end{array}$ & $\begin{array}{l}\text { R3-47 } \\
\text { S5-13 } \\
\text { S5-05 } \\
\text { R3-25 } \\
\text { R2-82 } \\
\text { R3-72 } \\
\text { H5-49 } \\
\text { R3-25 } \\
\text { R3-25 } \\
\text { R2-88 } \\
\text { R3-25 } \\
\text { R3-25 } \\
\text { R2-84 } \\
\text { R1-56 } \\
\text { S2-25 } \\
\text { G3-12 } \\
\text { R1-49 } \\
\text { R1-29 } \\
\text { S2-53 }\end{array}$ & $\begin{array}{l}1 \\
1 \\
1 \\
1 \\
1 \\
1 \\
1 \\
1 \\
1 \\
1 \\
1 \\
1 \\
1 \\
1 \\
1 \\
3 \\
1 \\
1 \\
1\end{array}$ & & & \\
\hline
\end{tabular}

For changes in distribution, please contact Tammy Rettkowski at 372-2559 or Linda Syverson on $376-0486$ 